\title{
A New Vehicle License Plate Correction Method Based on Sobel Operator and Priori Knowledge
}

\author{
Yong Wang ${ }^{1}$,Yan-Tao Chen ${ }^{2, *}$ Mao-Ning Wang ${ }^{3,{ }^{*}}$ \\ ${ }^{1}$ college of computer science, Sichuan University, Chengdu 610000, PRC \\ hfutwangyong@163.com
}

${ }^{2,3}$ Office of Academic Affairs, college of computer science, Sichuan University, Chengdu 610000, PRC

$2^{*}$ chenyantao@scu.edu.cn, ${ }^{3 *} 782402936 @ q q . c o m$

Keywords: License plate recognition; Tilt correction; Sobel operator; Affine transformation

\begin{abstract}
The tilted visual angel of the camera determines that incline will inevitably occur in vehicle license plate (VLP) recognition system. This will bring about difficulties to the following character segmentation and character recognition. Traditional correction methods can receive high accuracy hardly and require a large amount of calculation consumption. In response to this situation, this paper proposes a tilt correction algorithm based on Sobel vertical edges of the plate image and some priori information. Sobel operator is applied for vertical edge detection of VLP images, and statistical edge information is used to determine the horizontal deflection angel. Vertical tilt correction uses a priori knowledge that the characters have minimum width when they stand vertically, adjust the angle of the individual character to determine the vertical tilt angle. The experimental results verify that this proposed method can be easily implemented, and can quickly and accurately get the tilt angle, it also has strong robustness.
\end{abstract}

\section{Introduction}

Intelligent transportation gradually becomes the future of ground traffic with the rapid development of society. Automatic vehicle license plate(VLP) recognition technology is a key technology in the intelligent transportation system (ITS) .It has been widely used in urban parking zones, highway toll stations, vehicle violation detection , automatic registration of plate number and other fields . With the wide use of vehicles, government sectors have already payed more and more attention to the advanced, efficient, and accurate ITS . Recently, the necessity of VLP recognition has increased significantly. But because Cameras for photographing license plates are usually installed on the sides of the lane

in practical application, the shooting distance and shooting angle will cause that the VLP in the image frequently has some obvious and serious tilt[13]. The tilt will cause serious impact on the subsequent character segmentation and character recognition.

As far as tilt correction of the license plate region are concerned, various correction methods have been proposed. Methods commonly used in VLP correction are the Hough transformation and Radon transformation method .In[1-4], these papers proposed Hough transformation to estimate VLP position and calculate the tilt angle by using Different forms of Hough transformation. But in reality, Hough transform method based on Hough transform to determine inclination angle of the license plate frame; or extract license plate frame parameters by the Hough transform, and solve 4 vertices coordinates of the plate region, then correct the VLP image by the bilinear transformation. But the reality the license plates image contains noise, stains and other interference, plus the binaryzation of VLP images, making the plate frame not obvious, incomplete or cannot even see, resulting in dispersion peaks in the parameter space, lead to the detection effect is not ideal, and the amount of calculation is large.

In [5-9] ,Radon transform is used to project the image to 1-D space, and then according to the transform results using different methods to obtain the tilt angle of license plate. But the Radon 
transformation method need to rotate graphics and project edge points to ordinate direction.This method has an extremely heavy computational load and requires a lot of time because it needs to calculate the projection shape of each angle. It is difficult to achieve real-time requirements in the practical application.

In[10], a linear fitting method based on least square fitting with perpendicular offsets for correcting horizontal tilt.In vertical direction by using minimizing variance of coordinates of the projection points to correct vertical tilt angle. In[11], the author use K-L transformation to carry on tilt correction. In vertical tilt correction proposed namely K-L transformation, line fitting method based on K-means clustering and line fitting based on least square,a common failue of this paper is didn't prove the theory through a large number of experiments. In [12], Author put a brand-new perspective from color edge detection and the rotation of the projection to obtain the horizontal and vertical tilt angle of VLP. In [14] ,the tilt angle was detected according to the maximum value of the sum of the 4 maximum projection values, but it is also based on the linear features of the license plate frame.

As has been said, the above correcting tilt methods have the same drawbacks that the computational load is considerably heavy and the processing time is rather long, In addition, the correction accuracy is poor in the VLP tilt images.

In order to solve the issues, on the basis of Sobel operator and some priori knowledge, we proposes a new method for correcting a VLP tilt. correct VPL graphics from the horizontal and inclined directions respectively. In the horizontal tilt correction process, We using Sobel operator to detect graphics' vertical edge , after vertical edge detection using statistical method to obtain the horizontal declination angle. In the vertical tilt correction process, using VPL graphics the that have been corrected in horizontal direction ,delimiting graphics character outline ,applying priori knowledge that the character have a minimum width when the character is vertical. obtain each character's vertical deflection angle by correct characters respectively . Regard the majority angle as the vertical deflection angle. Affine transformation is inflicted on the rotated image and final correction image is obtained.

\section{Preliminary Knowledge}

\subsection{License Plate Tilt Mode}

There are three possible tilts modes of VPL[14]: horizontal tilt(see Fig.1),vertical tilt(see Fig.2), and hybrid tilt(see Fig.3). In summary, VPL correction including horizontal correction and vertical correction. In Fig.1,there is a horizontal tilt angle $\alpha$ between the horizontal axis $X$ and the tilt region principal axis $X^{\prime}$ and there are two cases of $\alpha$, once $\alpha$ is obtained ,so that the VPL can be corrected by rotate image at $-\alpha$.In Fig.2,The VPL slant along the perpendicular direction, vertical tilt is a kind of shear phenomenon of images, assume the vertical angle is $\beta$, we only do inverse affine transformation to complete the vertical tilt correction. Fig. 3 is the third tilt phenomenon including both horizontal tilt and vertical tilt, this is the most common tilt situation . Therefore, the key to correct VPL is how to obtain the deflection angle.
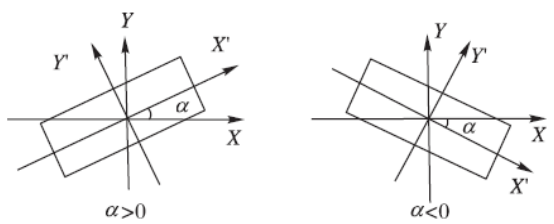

Fig.1 Horizontal tilt

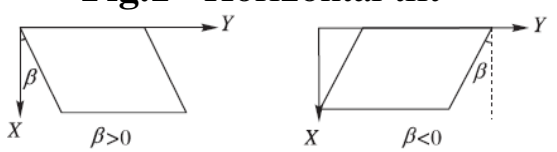

Fig.2 Vertical tilt 


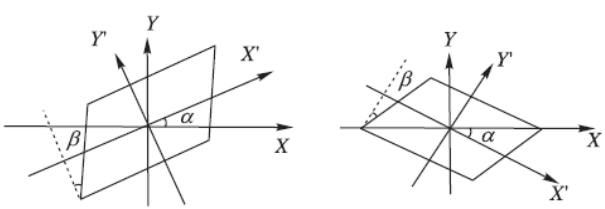

Fig.3 Hybrid tilt

\subsection{Vertical Edge Detection}

Sobel operator is a kind of first order operator , this operator according to the differential gray weighted neighborhood around the pixel points,judging whether the point is the extreme point , consider the point is the edge point if it is extreme point. Sobel operator is divided into horizontal and vertical two operator,were used to detect the vertical edges and horizontal edges of images, respectively. The advantages of the Sobel operator is easy and fast enough and can meet the requirement of real-time. Firstly algorithm maps the color space from RGB to Gray, use Sobel operator to detect vertical edge detection in horizontal correction phase. Suppose $I$ is the gray image matrix ,let $G_{y}$ be a gradient matrix:

$G_{y}=\left[\begin{array}{ccc}-1 & -2 & -1 \\ 0 & 0 & 0 \\ 1 & 2 & 1\end{array}\right]$

The vertical edge image $I_{b}$ can be expressed as :

$$
I_{b}=G_{y} * I
$$

\subsection{Affine Transformation}

The affine transformation is a linear transformation of two-dimensional coordinates to 2D coordinates.The license plate tilt problem can be seen as a kind of special affine transformation.An arbitrary affine transformation can be expressed as such form that multiplied by a matrix then plus a vector. Define the transformation matrix $A$ and translation matrix $\boldsymbol{B}$, affine transformation process can be written as:

$$
A=\left[\begin{array}{ll}
a_{00} & a_{01} \\
a_{10} & a_{11}
\end{array}\right], B=\left[\begin{array}{l}
b_{00} \\
b_{10}
\end{array}\right],\left(\begin{array}{l}
x^{\prime} \\
y^{\prime}
\end{array}\right)=A \cdot\left(\begin{array}{l}
x \\
y
\end{array}\right)+B
$$

Among them, the horizontal angle of the license plate is $\alpha$ (suppose counterclockwise rotation correction is positive), the counterclockwise rotation matrix $R(\alpha)$ which rotates around the origin point is :

$$
R(\alpha)=\left[\begin{array}{ccc}
\cos \alpha & -\sin \alpha & 0 \\
\sin \alpha & \cos \alpha & 0 \\
0 & 0 & 1
\end{array}\right]
$$

Vertical tilt is the shear deformation in the $Y$ axis direction (suppose inclination angle relative to the $Y$ positive direction is $\beta$ ), the shear transform matrix :

$S(\beta)=\left[\begin{array}{ccc}1 & 0 & 0 \\ \tan \beta & 1 & 0 \\ 0 & 0 & 1\end{array}\right]$

Because of the multiplication of matrix does not meet the exchange rate, the VLP correction process can divided into two steps, the first step is horizontal correction, then do the vertical correction. Transformation matrix $\mathrm{W}$ can express as :

$W=S(\beta) \times R(\alpha)$

we can obtain the finally transformation matrix by the horizontal tilt angle $\alpha$ and the vertical tilt 
angle $\beta$. Complete license plate correction work by using above formula (6).

\section{License Plate Correction Algorithm}

\subsection{Calculating the Horizontal Tilt Angle}

License plate contains abundant vertical edges, but the background contains little. The summation of gray value reach to maximum in the character area when a VLP in a horizontal position. In this paper, using Sobel horizontal operator to obtain vertical edges of the VPL, then pinpoint the approximate location of the character area from license plat. Proposed a new method to obtain the horizontal tilt angle of the license plate by counting the vertical edge information.

The specific steps to calculate the horizontal angle of the license plate described as follows:

Step1:Transfer the VLP to gray one,then obtain vertical edge image $I_{b}$ by using Sobel horizontal operator.

Step2:Select the perpendicular bisector of $I_{b}$,suppose column number is Col .Draw a rectangle $C$, take the $C o l$ as the center . The width of $C$ is $\frac{1}{8} I_{b}$ width.

Step3:Suppose matrix $\left(a_{1}, a_{2}, \ldots \ldots a_{i}\right), i$ is row numbers of $C$, means the gray value of each line .Suppose :

$$
\operatorname{MaxGap} 1=\underset{0 \leq j \leq \frac{i}{2}}{\operatorname{Max}}\left(a_{j+1}-a_{j}\right) \quad \operatorname{MaxGap} 2=\underset{\frac{i}{2}<j \leq i}{\operatorname{Max}}\left(a_{j+1}-a_{j}\right)
$$

MaxGap1, MaxGap2 are two rows which the summation of gray value change most in left and right sides divide by the middle row of $C$.

Draw a rectangle $H$, set the height to $h$, and the length is len $H$ :

$$
\begin{aligned}
& h=(\text { MaxGap2 - MaxGap1) } \\
& \text { lenH }=4.5 \times h
\end{aligned}
$$

According to the experience, rectangle $H$ is the character area of the VLP.

$P$ is the point regarded as the center of $H$.

$$
P:\left(\operatorname{col}, \frac{\text { MaxGap1+MaxGap2 }}{2}\right)
$$

Step4:Rotate $I_{b}$ around the point $P$ regarding $1^{\circ}$ as the step length .Set $\left[-30^{\circ}, 30^{\circ}\right]$ to be the rotate range . Count the summation gray value of each row in $H$ after each rotation. Where $f\left(x_{i}, y_{i}\right)$ is the gray value of the pixel $\left(x_{i}, y_{i}\right)$, this can be represented by a vector $\mathrm{B}$ :

$$
\begin{aligned}
& B:\left(b_{1}, b_{2}, b_{3} \cdots \cdots, b_{i}\right), i=60 \\
& b_{i}=\sum_{y_{i}=1}^{\operatorname{lenH}} \sum_{x_{i}=1}^{h} f\left(x_{i}, y_{i}\right)
\end{aligned}
$$

Set :

MaxSobel $=\operatorname{Max}\left(b_{1}, b_{2}, b_{3} \cdots \cdots b_{i}\right)$

(12) The corresponding rotation angle of MaxSobel is the horizontal tilt angle .

\subsection{Vertical Tilt Correction}

In general, the license plate will appear the vertical inclination and horizontal tilt at the same time. Vertical tilt is a special shear phenomenon.We will complete vertical correction after horizontal correction. This paper presents a new method for vertical correction. The method with good anti-interference and simple calculation,and does not depend on the VLP frame feature.

We can easily find that the vertical tilt angle of characters is same with the entire VLP. The characters in license plate can be divided into two categories: The first kind is such characters which 
the width is not consistent between the top and below , and have no vertical line such as $\mathrm{A}, 7,4, \mathrm{~V}, \mathrm{Y}$...Another class is these characters which have roughly same width or with right angle side such as D,8,1,2,L,P...

According to the priori knowledge, the first kind of characters' width are minimal within a certain amount of deflection range ,but the second type of characters have a minimum width when a character is not vertically tilted.See Fig.4, the width of character ' $S$ ' is 105 pixels when character have a vertical slope Angle, but when the ' $S$ ' in the vertical position ,character have minimum width 97 pixels.

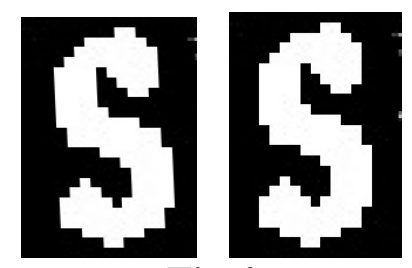

Fig.4

Thus, in this paper, the vertical correction method based on priori knowledge above. At first , license plate was binarized by using local threshold method. extract each character contour from binary image, discard the first and last contour because the robustness of these contour is largely influenced by background, then correct these contours, making the width of the outline to be minimal, calculate the modal number of these tilt angles. Finally, regard the modal number as the vertical deflection angle of the license plate.

After got the vertical angle, adjust VLP by affine transformation, take up-left corner as the origin point. Suppose the vertical deflection angle is $\beta$. As shown in Fig.5,the coordinate $A(x, y)$ can be translated to $A^{\prime}\left(x^{\prime}, y^{\prime}\right)$ after affine transformation. This counterclockwise rotation around the vertical direction is known as the shear transform. Cooridinate transformation can be expressed as (13):

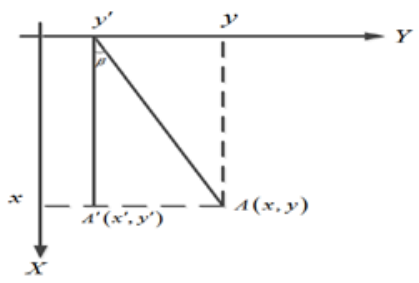

Fig.5 Shear transformation model

$$
\left\{\begin{array}{l}
x^{\prime}=x \\
y^{\prime}=y+x \tan \beta
\end{array}\right.
$$

Specific steps for the vertical correction method is:

Step1: Extra rectangle $H$ from the license plate that have been horizontal-corrected. Using OSTU binary algorithm to process $H$.In which the background is black (gray value is 0 ) and the characters are white (gray value is 255).

Step2: Extra contours from the binary license plate.

Step3: Find the smallest rectangles that can surround each profile, the width of the rectangle is the width of each contour.

Step4: Adjust each contour, taking the up-left corner as the origin point and regarding $1^{\circ}$ as step length ,set $\left[-30^{\circ}, 30^{\circ}\right]$ to be the adjust range. Write down the rotation angle of each contour, calculate the modal number of these angle. When have more than one modal number, calculating the average angle. Regard the mode as the vertical deflection angle of the license plate. Finally, complete the vertical correction for the license plate. 


\section{Experiment}

\subsection{Horizontal correction}

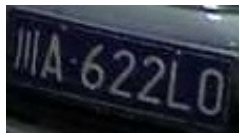

(a)

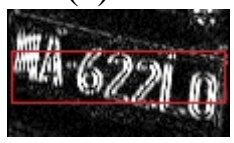

(c)

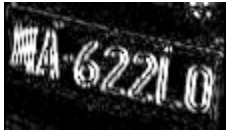

(b)

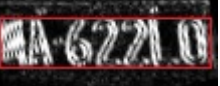

(d)

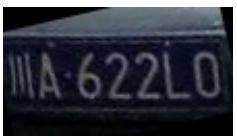

(e)

Fig.6 Horizontal correction process

Fig.6 shows the process of the license plate horizontal calibration, (a) is the original license plate image,(b) is vertical edge image, processing by Sobel horizontal operator. In the intermediate position of (c) there is a red rectangular box. Rotating the vertical edge image and count the summation gray value in the red rectangle area. Obtain the Maximum value when rotate to the position like (d)

isrbBat,(en) ingle fiøal desult after horizontal correction.

\subsection{Vertical correction}

\section{MA-622L0}

(a)

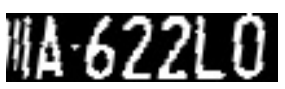

(d)

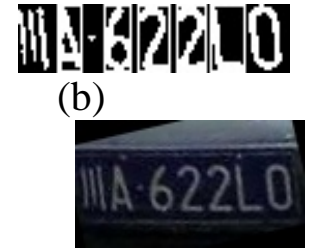

(e)

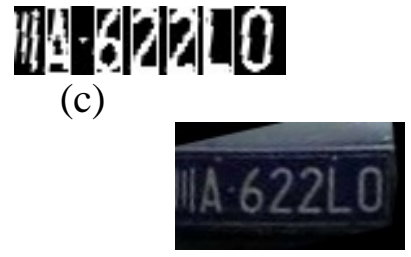

(f)

Fig.7 Vertical correction process

Process the VPL after horizontal correction, gray image is changed to binary image by OSTU algorithm as (a) shown. (b) identifies the surrounding rectangle of each profile, ignore the fore and finally two rectangles. According to the priori knowledge, obtain minimum width of each contour by correcting these contours respectively. The original width of the first and second contours are 15 pixels, when the deflection range between $\left[6^{\circ}, 11^{\circ}\right]$,the width of first two contours is the smallest; the original width of the third and fourth profiles is 15 pixels, width of these two contours reach to the 12 pixels when the deflection be $8^{\circ}, 9^{\circ}$; the original width of the fifth profiles is 15 pixels ; width of this contour reach to minimum 11 pixels when the deflection be $-9^{\circ}$. So take $-9^{\circ}$ as vertical deflection angle of license plate. The result after affine transformation is (f). Fig.7 shows the whole vertical correction process.

\subsection{Experiment result}

The experiments are all performed in Opencv on a PC with a Core 2.4GHz processor and 2GB RAM, running with CWindow7.Using Hough transformation, Radon transformation and adjustment method of this paper to adjust VLP images. We compare the horizontal correction experiment result using two specific images.The correlation indexes in Table 1 are in accordance with the results in Figs.9-11.

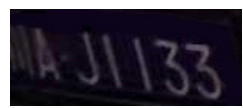

(a)original image $\mathrm{A}$

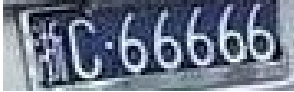

(b) original image $\mathrm{B}$

Fig.8 Two example image 


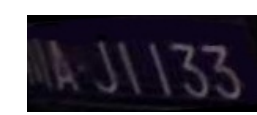

(a)original image $\mathrm{A}$

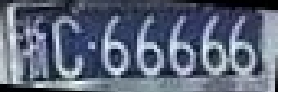

(b) original image $\mathrm{B}$

Fig.9 Horizontal tilt correction of Hough transformation

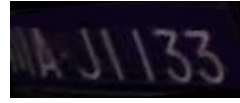

(a)original image $\mathrm{A}$

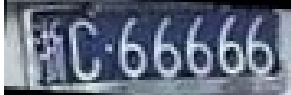

(b) original image $\mathrm{B}$

Fig.10 Horizontal tilt correction of Radon transformation

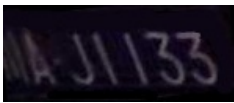

(a)original image A

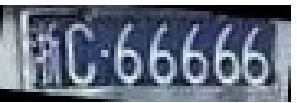

(b) original image $\mathrm{B}$

Fig.11 Horizontal tilt correction of the proposed method Table 1. The results of two examples VLP

\begin{tabular}{|c|c|c|c|}
\hline Method & Index & image $A$ & image $B$ \\
\hline \multirow{2}{*}{$\begin{array}{c}\text { Hough } \\
\text { Transformation }\end{array}$} & Deviation angle & $3.2^{\circ}$ & $1.4^{\circ}$ \\
\hline & Time & $0.095 \mathrm{~s}$ & $0.089 \mathrm{~s}$ \\
\hline \multirow{2}{*}{$\begin{array}{c}\text { Radon } \\
\text { Transformation }\end{array}$} & Deviation angle & $1.5^{\circ}$ & $1.7^{\circ}$ \\
\hline & Time & $0.102 \mathrm{~s}$ & $0.095 \mathrm{~s}$ \\
\hline Proposed & Deviation angle & $0.6^{\circ}$ & $0.5^{\circ}$ \\
\hline Method & Time & $0.032 \mathrm{~s}$ & $0.036 \mathrm{~s}$ \\
\hline
\end{tabular}

The experiment also selected 100 images totally with different inclination degree. These images contain no border VLP images and images with vague frame. At the Horizontal correction stage, the average processing time (s) and average tilt angle $\left({ }^{\circ}\right)$ of the three methods are shown in Table 2 .

Table 2. The average tilt angle and process time

\begin{tabular}{|c|c|c|}
\hline Method & Index & Result \\
\hline \multirow{2}{*}{ Hough Transformation } & Average deviation angle & $2.9^{\circ}$ \\
\cline { 2 - 3 } & Average time & $0.094 \mathrm{~s}$ \\
\hline \multirow{2}{*}{ Radon Transformation } & Average deviation angle & $2.3^{\circ}$ \\
\cline { 2 - 3 } & Average time & $0.100 \mathrm{~s}$ \\
\hline \multirow{2}{*}{ Proposed Method } & Average deviation angle & $0.6^{\circ}$ \\
& Average time & $0.030 \mathrm{~s}$ \\
\hline
\end{tabular}

As shown in the Table2. In horizontal tilt correction experiment, we compared the three methods to each other. Hough transformation is suitable for these images which have the obvious frame, but the tilt angle of the images which have vague frame cannot be obtained accurately. Radon transform has a relatively high accuracy rate, however the running time is longer, in practical engineering, it is very difficult to guarantee the requirement of real time. Our algorithm is simple to operate, with high 
accuracy calibration and fast speed.

At vertical tilt correction stage, we using 90 pictures which after horizontal correction, the image examples like Fig. 11 and Fig 12 .Comparing with manual adjustment, set $\left[-2^{\circ}, 2^{\circ}\right]$ be the tolerance error range, the result is shown in Table 3 :

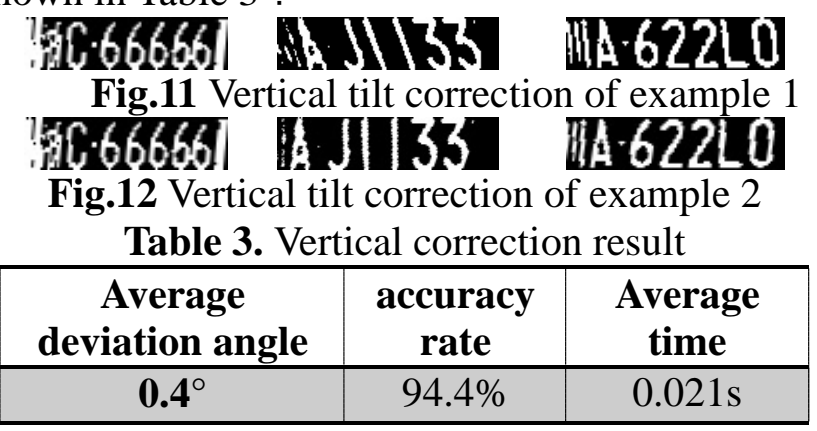

\section{Conclusion}

Faced with the unsatisfactory situation that using traditional license plate correction algorithms. After observe the license plate image carefully, using license plate itself morphological characteristics combined with Sobel horizontal operator and some priori knowledge. Two solutions were proposed for the problem of the horizontal and vertical tilt of the plate respectively. The proposal method improved the precision of license plate tilt testing, reduce the testing time. Compare with the Hough transform and Radon transform in the horizontal tilt correction experiment, the result shows that our algorithm in terms of correcting time or accuracy are better than two conventional correction method.

In the vertical correction phase, experiment used 90 test images which have been corrected in horizontal direction, the vertical correction algorithm based on a priori knowledge can ensure a high accuracy and can finish the work in a short time as the result shown.

\section{Acknowledgments}

This work was supported by Science and Technology Achievement Transformation Project of Sichuan Province, PRC (No.14CG0059) and Fundamental Research Funds for the Central Universities,PRC(No.2015SCU11071).

\section{References}

[1] H.A. Hegt, R.J. Haye, N.A.Khan.: A High Performance License Plate Recognition System. In: Proceedings of the IEEE International Conference on Systems, Man, and Cybernetics, IEEE Press, vol. 5, pp. 4357-4362, (1998).

[2] C. Y. Wen, C. C. Yu, Z. D. Hun. :A 3D Transformation to Improve the Legibility of License Plate Numbers. J. Journal of Forensic Sciences, vol. 47, no. 3, pp. 578-585, (2002).

[3] Y. T. Ching. :Detecting Line Segments in an Image A New Implementation for Hough Transform. J. Pattern Recognition Letters, vol. 22, no. 3, pp. 421-2429, (2001)

[4]T.F. Ma, Y.G.Zheng, W.D.Zhao . :Algorithm based on edge detect ion and Hough transform at ion for segmentation of plate characters. J. Journal of System Simulation,vol.18(Suppl1),pp:391-392,(2006)

[5]L.P. Gong, Y.P. Bai.: Application of Radon transformation to license plate slant correction. J. Journal of Test and Measurement Technology.vol.23,no.5,pp: 452-456.(2006)

[6] Rajiv Kapoor,Deepak Bagai,T. S. Kamal. A new algorithm for skew detection and correction. J. Pattern Recognition,vol.25,no.11,pp:1215-1229,(2004)

[7] L.G. Yang, X.H. Zhang,L.Y. Li.: Study on method of correcting lean license plate characters. J. 
Chinese Journal of Scientific Instrument.vol.25,no.4, pp.696-697.(2004)

[8]X.D. Jia, W.J. Li,H.J. Wang.:Novel approach for vehicle license plate tilt correction based on Radon transform. J. Computer Engineering and Applications, vol.44 ,no.3, pp.245-248.2008.

[9] L.X. Gong, H.P. Hu ,Y.P. Bai.: Vehicle license plate slant correction based on mathematical morphology and radon transformation.In:5th International Conference on Natural Computation(ICNC). pp:3457-3461;Yantai (2010)

[10]Kaushik, D., Andrey, V., Jung, W.K.: Vehicle license plate tilt correction based on the straight line fitting method and minimizing variance of coordinates of projection points. J. International Journal of Control, Automation, and Systems. vol.8,no.5,pp:975-984.(2010)

[11]M.S. Pan., Q. Xiong., J.B. Yan.A New Method for Correcting Vehicle License Plate Tilt. J. International Journal of Automation and Computing.vol.6,no.2,pp: 210-216 .(2009).

[12] W.J. Li., D.Q.Liang., L.Y. Cui, et al. :A Novel approach for vehicle license plate tilt correction. J. In: Information and Control, vol.33,no.2,pp:231-235.(2004) .

[13]Guillou, E, Meneveaux, D,Maisel, E,et a1.:Using vanishing points for camera calibration and coarse 3D reconst ruction from a single image. J. The Visual Computer,vol.16,no.7,pp:396-410,(2000).

[14] W. J. Li, D. Q. Liang, X. N. Wang, D. Yu. Character Segmentation for Degraded License Plate. J. Journal of Computer-Aided Design \& Computer Graphics, vol.16, no.5, pp.697-700, 2004. 